\title{
An Atomistic Study of Copper Extrusion in Through-Silicon-Via Using Phase Field Crystal Models
}

\author{
Zhiheng Huang ${ }^{1 *}$, Jinxin $\mathrm{Liu}^{2}$, Paul P. Conway ${ }^{3}$, Zhuojun $\mathrm{Hu}^{2}$ and Yang Liu ${ }^{4 *}$ \\ ${ }^{1}$ School of Materials Science and Engineering, Sun Yat-sen University, Guangzhou 510275, China \\ ${ }^{2}$ School of Physics, Sun Yat-sen University, Guangzhou 510275, China \\ ${ }^{3}$ School of Mechanical, Electrical and Manufacturing Engineering, Loughborough University, Loughborough LE11 3TU, UK \\ ${ }^{4}$ School of Electronics and Information Technology, Sun Yat-sen University, Guangzhou 510006, China \\ *Corresponding author, email addresses: hzh29@mail.sysu.edu.cn (Z. Huang), liuy69@mail.sysu.edu.cn (Y. Liu)
}

\begin{abstract}
Three-dimensional system integration using $\mathrm{Cu}$ through-silicon-via (TSV) technology enables vertical interconnection of stacked dies. However, the large statistical distribution of plastic $\mathrm{Cu}$ extrusion, also known as $\mathrm{Cu}$ pumping, presents a serious reliability concern. Traditional finite element method (FEM) based thermomechanical modeling that neglects microstructure has been extensively attempted in order to identify the root cause of the extrusion, which yet remains unknown. This study utilizes recently developed phase field crystal (PFC) models, which resolve systems on atomic length scales and diffusive timescales, to capture the creation, destruction, and interaction of defects in polycrystalline $\mathrm{Cu}$ TSV structures and thereby elucidate the atomistic mechanisms of the $\mathrm{Cu}$ extrusion. The governing kinetic equation of the PFC model is first solved using FEM to generate $\mathrm{Cu}$ grains with an atomic resolution in TSVs by referring to experimental EBSD images. A shearing term is then added to the governing equation to simulate TSV deformation under shear strain. The solidification process at the atomistic scale is simulated to prepare polycrystalline TSV samples. Rotation and coalescence of grains with low mis-orientations are observed in solidification. The application of shear strain to the polycrystalline TSVs reveals the movement of defects at the atomistic scale. The defects diffuse through grain boundaries and aggregate at the edges of TSVs, where the defects become immobile. The process of rotation and coalescence of grains is found to be accelerated under the shear strain. The simulation results also suggest that the geometry of the TSVs is an important factor controlling the behavior of defect diffusion and microstructures in TSVs, and thus the mechanical behavior of TSVs.
\end{abstract}

\section{Introduction}

Three-dimensional integration is an emerging technology that enables vertical interconnection of stacked dies, meeting the demands for smaller scale, higher density and shorter signal paths of microelectronic devices [1]. As a key approach of three-dimensional interconnection schemes, electronic packaging using Through-Silicon-Vias (TSVs) has attracted widespread interest [2]. However, the plastic $\mathrm{Cu}$ extrusion in TSVs, also known as $\mathrm{Cu}$ pumping, presents a serious reliability concern. The effects of annealing [3] and thermal cycling [4] on thermal stress have been studied, attempting to identify the root cause of the $\mathrm{Cu}$ extrusion.

Reliability complications in TSVs typically arise either from stress-related or electrical sources, and are often convoluted with microstructural effects. The stresses induced near the surface of $\mathrm{Si}$ next to the $\mathrm{Cu}-$ filled TSVs have an adverse impact on the electrical performance of devices in the immediate neighborhood of the TSVs due to induced piezoresistivity, which results in degradation of carrier mobility [5]. This necessitates a keep-out zone (KOZ) in the $\mathrm{Si}$, typically a few micrometers in width, in the immediate vicinity of each TSV, where active devices cannot be placed. Various approaches have been utilized to understand the nature of these stresses. Three main techniques that been used to measure the stress state in TSVs are wafer curvature method [6-8], micro-Raman spectroscopy [8,9] and X-ray diffraction $[10,11]$ based techniques. Although the synchrotron X-ray micro-diffraction is by far the most powerful technique for stress analysis, it is the most expensive and requires access to a synchrotron radiation source [11]. Furthermore, none of these aforementioned techniques can directly measure stress in $\mathrm{Si}$ or $\mathrm{Cu}$. Therefore, a complete picture of the stress distribution in TSVs is still unavailable. However, this information is critical to elucidate the root cause for the metal pumping phenomenon.

The challenge of managing mechanical stress is not new, and a number of simulators do exist and have a long track record of use in the electronics industry. Most of the proven simulators are based on finite element method (FEM), or derivatives of that class of modelling technique. The established FEM stress simulators have typically been used for addressing the traditional chippackage interactions, and have therefore mostly modelled physical deformations, such as cracking, delaminating, or fracturing [12]. In this class of analyses Si dies and TSV fillers are typically modelled as monolithic bricks and isotropic material models based on empirical equations are used, even though it has been known since 1934 that crystalline materials deform plastically by the slip of dislocations on discrete slip systems [13].

Finite element based modelling work has also reported the linkage between the microstructure of copper grains and the stress in $\mathrm{Cu}$ TSVs. The results from a linear elastic mechanical model clearly demonstrate that the stress distribution is rather heterogeneous inside the TSV filler, considering the anisotropy of the elastic compliance tensor of copper. Depending on the texture, morphology and distribution of the copper grains, stress concentrations may occur at the grain boundaries (GBs) [14]. Elastoplastic models have also been conducted directly on copper grain structures with the aim of explaining the formation of $\mathrm{Cu}$ extrusion [15]. However, the drawbacks 
of this kind of model are clear. First, although the mechanical analysis is conducted directly on copper microstructure, the microstructure is assumed to remain unchanged during the mechanical loading process. Second, the plastic deformation mechanisms at the atomic scale, e.g. the motion of dislocations, have not been considered. However, the dislocation involved processes such as recovery, recrystallization and grain growth have been reported to occur in electrodeposited copper and can significantly influence its mechanical properties. Recrystallization has also been suspected to account for the copper-pumping phenomenon $[7,16]$. Considering the limitation of the current modeling techniques, more robust simulation methodologies that take into account the polycrystalline nature of $\mathrm{Cu}$ TSVs and their complex deformation and stress relaxation mechanisms are needed [16].

A physically based crystal plasticity finite element (CPFE) method is relevant in order to address the polycrystalline nature of $\mathrm{Cu}$ TSVs and their complex deformation and stress relaxation mechanisms. The result from a unidirectional compression on a copper single crystal using the CPFE method has been reported [17]. Although the mechanical boundary conditions and the material are both different from the real $\mathrm{Cu}$ TSV structures, the result highlights the usefulness of the CPFE method in capturing atomistically-informed deformation and deformation induced crystallographic orientation evolution. The body centered cubic (BCC) tungsten single crystal subjected to uniaxial loading has also been recently studied [18].

More recently, a new class of phase field model has been developed, called the phase field crystal (PFC) model, which describes the thermodynamics and kinetics of phase transformations through an atomically varying order parameter field that is loosely connected to the atomic density field [19,20]. PFC models naturally capture most of the salient physics of nucleation, polycrystalline solidification, GBs [20,21], and solidification in multicomponent and multiphase systems $[22,23]$. In addition, PFC models also capture, in the context of a single order parameter, elasticity and plasticity phenomena relevant to solid-state processes such as dislocation source creation, dislocation stability [24,25], and creep [26]. To study the $\mathrm{Cu}$ extrusion phenomenon, mechanical loads have to be applied on such samples with desired grain structures. Since the PFC method does not model a solid-vacuum interface, traction boundary conditions in the PFC model using a penalty term are introduced. In deformation simulations, dislocation creation and annihilation are emergent characteristics of the PFC model. Therefore, applying the PFC model to the TSV filler allows the investigation of the dislocation dynamics responsible for the metal extrusion or intrusion problem. The recorded dislocation dynamics can also be used to formulate dislocation-based constitutive laws for the CPFE method. In addition, progress has been made to couple the atomic-scale PFC density field to order parameters that describe ferromagnetic and ferroelectric ordering [27]. As such, the PFC models can be used to study the role of external magnetic or electric fields on the evolution of atomic scale defect structures on diffusion time scales. This provides an opportunity to study electromigration related reliability issues of TSVs using the PFC models.

In this study, the PFC model is utilized to simulate the polycrystalline $\mathrm{Cu}$ in TSV at atomic scales under shear strain. The rest of the paper is organized as following. First, the PFC model is briefly reviewed, and then the preparation of $\mathrm{Cu}$ TSV samples is introduced. Second, the atomistic behavior of polycrystalline $\mathrm{Cu}$ TSVs under shear strain is presented. Finally, the geometry effect on the deformation behavior of $\mathrm{Cu}$ TSVs is discussed.

\section{Modelling Approach}

In the PFC model, the free energy function of a periodic system is minimized by an order parameter corresponding to local-time-averaged atomic density. The dimensionless free energy function is written as [20]

$$
F=\int d V\left[\frac{\rho}{2}\left(r+\left(1+\nabla^{2}\right)^{2}\right) \rho+\frac{\rho^{4}}{4}\right]
$$

where $r=-0.25$ is a temperature-related parameter and $\rho$ is the order parameter. The governing equation of the PFC model is

$$
\frac{\partial \rho}{\partial t}=\nabla^{2} \frac{\delta F}{\delta \rho}+\xi=\nabla^{2}\left[\left(r+\left(1+\nabla^{2}\right)^{2}\right) \rho+\rho^{3}\right]+\xi
$$

where $\xi$ is a Gaussian random noise term that has been neglected in this study. This study focuses on two dimensional modelling and the stable solution of the governing dynamic equation is a triangular lattice. Considering the $\{111\}$ plane in $\mathrm{FCC} \mathrm{Cu}$, the periodic triangular state is set as

$$
\rho(x, y)=\bar{\rho}+A\left[\cos (q x) \cos \left(\frac{q y}{\sqrt{3}}\right)-\frac{1}{2} \cos \left(\frac{2 q y}{\sqrt{3}}\right)\right]
$$

where $\bar{\rho}=0.285, q=\frac{\sqrt{3}}{2}$ and $A=0.47034$.

The TSVs are initialized to contain only the liquid phase in the simulations. The solidification process occurs when randomly distributed crystal nuclei are put inside the TSVs and TSVs composed of polycrystalline $\mathrm{Cu}$ structures, hereinafter referred to as TSV samples, are formed after the solidification completes.

To study the mechanical behaviour of the $\mathrm{Cu}$ TSVs under shear strain, the TSV samples are placed in contact with a pool of liquid on both the top and bottom sides. The free energy function is modified by adding a shearing term to simulate the application of a shear strain [28]

$$
\begin{gathered}
F_{\text {shear }}=\int d x d y\left[G(y-d)\left(\rho(x, y, t)-\rho_{0}(x-v t, y)\right)^{2}\right. \\
\left.+G\left(y-L_{y}+d\right)\left(\rho(x, y, t)-\rho_{0}(x+v t, y)\right)^{2}\right] \\
G(y)=\exp \left(-y^{2} / \sigma^{2}\right) / \sqrt{2 \pi \sigma^{2}}
\end{gathered}
$$

where $\rho_{0}$ corresponds to the analytical solution of the governing equation for a perfect single crystal and $G(y)$ is a normalized Gaussian function controlling the position in the $y$-direction where the shear strain is applied. 
As shown in Fig. 1, TSVs of three geometries, i.e. a rectangle, a trapezoid and an hourglass, are considered. Noted that the units for both the space and time are dimensionless in this study. The models are implemented in an integrated modelling platform combining the advantages of both MATLAB and COMSOL Multiphysics.

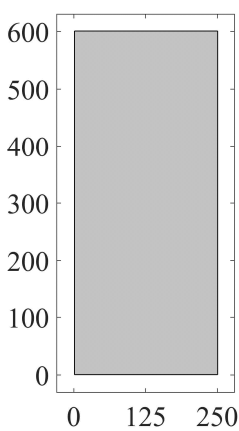

(a)

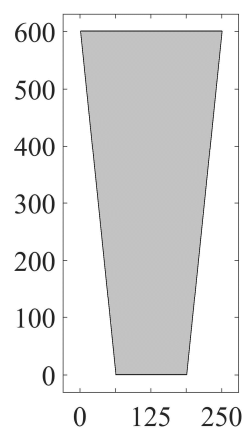

(b) (c)

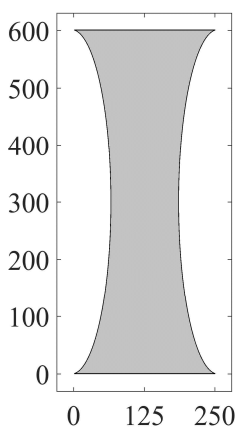

Fig. 1 Geometries of the TSVs: (a) A rectangle, (b) a trapezoid, and (c) an hourglass. Note that the units in both the $x$ and $y$-directions are dimensionless.

\section{Results and Discussion}

Preparation of Polycrystalline TSV Samples

Fig. 2 shows the snapshots of grain structure in the rectangular TSV during solidification at $t=50$ and 300 . The randomly located and oriented crystal nuclei are first placed in the TSV by referring to experimentally characterized grain structures in $\mathrm{Cu}$ TSVs using the EBSD technique [3]. Note that the initial configurations of the nuclei in TSVs of other geometries are kept the same as in the rectangle one, although some of the nuclei may be removed to adapt to the specific geometry.

Reorientation of the grains during solidification is observed as shown in the insert of Fig. 2. The orientations of grains 1 and 2 are illustrated by the dash and solid lines, respectively. It has been found that the orientation mismatch between the two grains decreases with time, i.e. from $8.1^{\circ}$ at $t=50$ to $5.1^{\circ}$ at $t=300$, showing a tendency towards grain coalescence. Thus, a low-angle GB is eventually formed between grains 1 and 2 . Different from the situation of grains 1 and 2, the misorientation between grains 3 and 4 slightly increases from $15.7^{\circ}$ to $16.1^{\circ}$ during the same period. The variations of the misorientation between other adjoining grains are also measured. In general, adjoining grains initially with a small mis-orientation tend to merge, while those with a larger mis-orientation only slightly change their orientations.

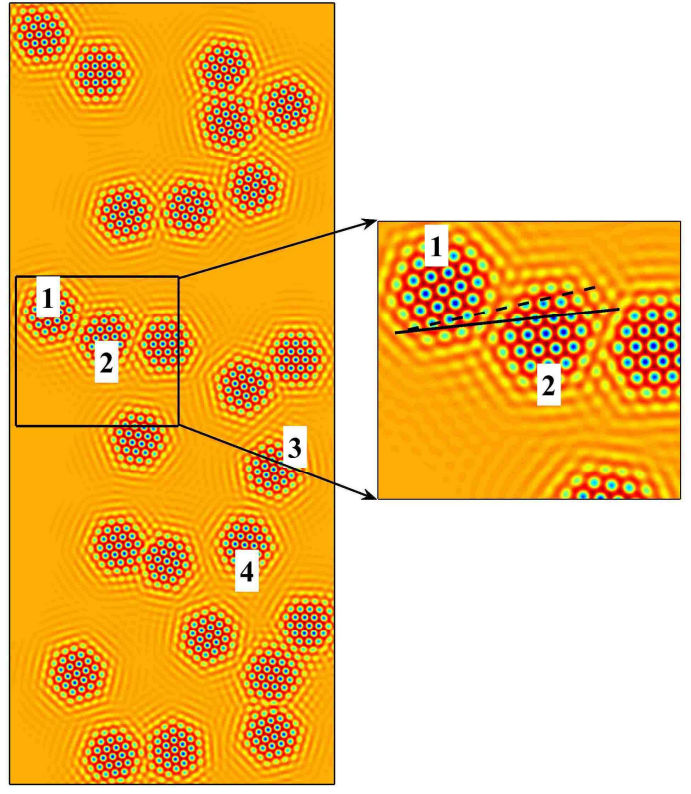

(a)

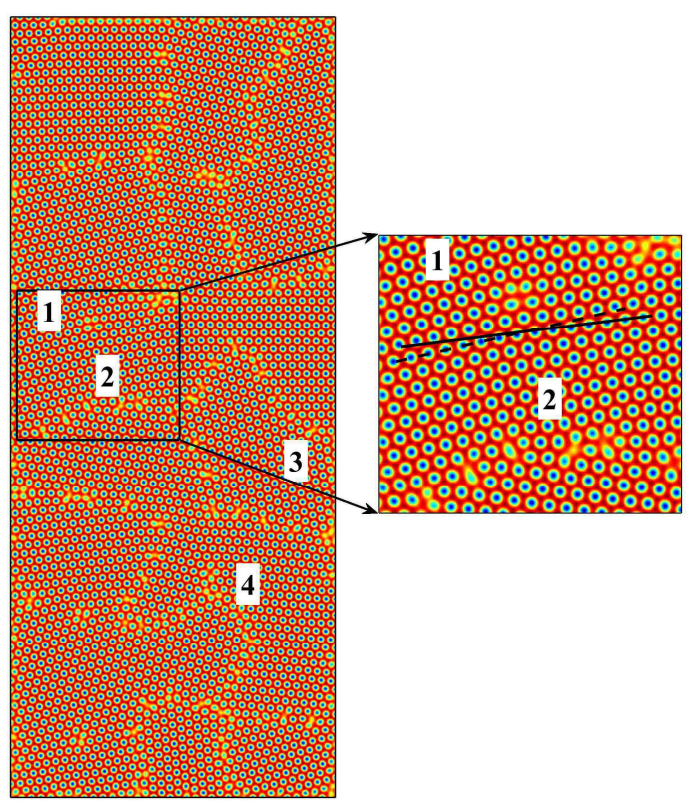

(b)

Fig. 2 Atomistic arrangements in the rectangle TSV at (a) $t=50$, and (b) $t=300$. The dash and solid lines show the orientation of grains 1 and 2 , respectively.

Fig. 3 plots liquid fraction versus time during solidification in the TSVs with different geometries. A threshold value of the order parameter $\rho$ is carefully chosen to identify the liquid phase. The result in Fig. 3 suggests that the effect of the TSV geometry on the solidification rate is not evident. The solidification rate decreases with time. At the beginning of the solidification, the grains are surrounded by a large amount of undercooled liquid, thereby receiving a sufficient liquid supply. As the solidification proceeds, the crystal grains grow and contact with either other grains or the edge of the TSV. As a result, the amount of remaining undercooled liquid decreases, leading to a lower 
solidification rate. Noted that the solidification rate reduces to zero at around $t=300$, indicating the end of the solidification process with the formation of a polycrystalline $\mathrm{Cu}$ TSV. Therefore, $t=300$ is chosen as the start time to apply the shear strain.

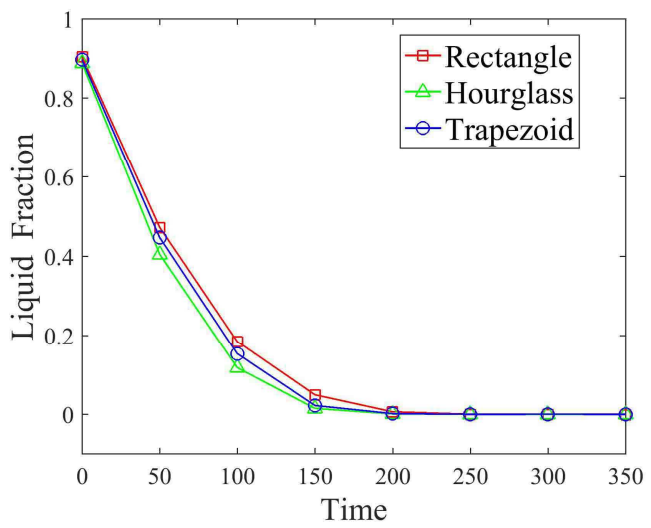

Fig. 3 Plots of liquid fraction versus time in TSVs with different geometries.

\section{Application of Shear Strain}

Fig. 4 illustrates the atomistic arrangements in the rectangular TSV under the shear strain. With the shear strain applied, grain rotation and grain coalescence are both observed. For example, the grain marked by the dash line in Fig. $4 \mathrm{~b}$ rotates $1.6^{\circ}$ to a new orientation indicated by the solid line in Fig. 4c from $t=2000$ to $t=10000$. Table I lists the orientation and size of the labelled grains in Fig. 4a. At $t=0$ of the loading stage, grains 3, 4, 5 and 6 are at approximately the same orientation because of the grain reorientation during the solidification stage. Those four grains, labelled with CG1 in Fig. 4b, gradually coalesce to form a larger grain as a result of grain rotation under the shear strain. The same phenomenon has been observed in a previous PFC simulation [29]. Grain coalescence between adjoining grains with a relatively larger mis-orientation is also observed. For example, because the orientation of CG1 dominates in the local area, grains 1 and 2, with a mis-orientation of approximately $37^{\circ}$ and $25^{\circ}$ with CG1 respectively, rotate to adjust their orientations and coalesce with CG1 to form an even larger grain labelled with CG2 in Fig. 4c at $t=$ 10000. Since grain rotation and coalescence occur during the solidification stage, the application of shear strain only accelerates such phenomena.

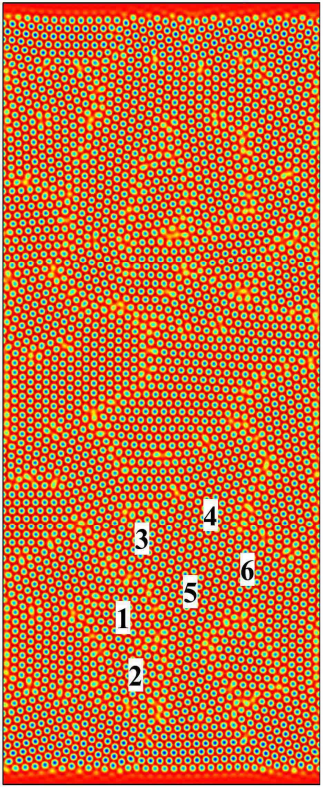

(a)

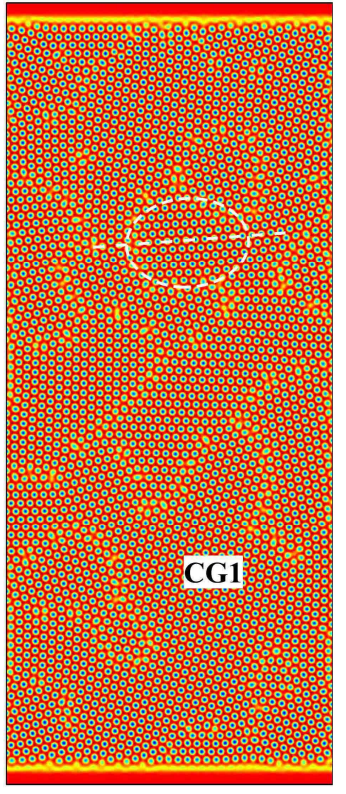

(b)

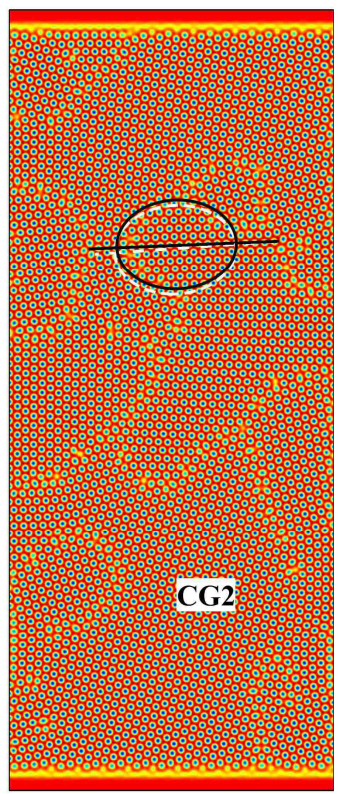

(c)

Fig. 4 Atomistic arrangements in the rectangle TSV under shear strain at (a) $t=0$, (b) $t=2000$, and (c) $t=10000$. The dash circle in (b) outlines the original shape of the grain. The solid circle in (c) outlines the shape of the grain after deformation, with the original shape superimposed.

Table I The orientation and diameter of grains 1-6

\begin{tabular}{|c|c|c|}
\hline Grain \# & Orientation & Diameter \\
\hline 1 & $56.9^{\circ}$ & $\sim 40$ \\
\hline 2 & $44.3^{\circ}$ & $\sim 40$ \\
\hline 3 & $19.5^{\circ}$ & $\sim 50$ \\
\hline 4 & $17.7^{\circ}$ & $\sim 50$ \\
\hline 5 & $18.0^{\circ}$ & $\sim 45$ \\
\hline 6 & $22.6^{\circ}$ & $\sim 50$ \\
\hline
\end{tabular}


In addition, the application of the shear strain to the TSVs causes the motion of the defects present in the GBs. The behaviour of the defect motion in the rectangular TSV is demonstrated in Figs. 5 and 6. Fig. 5 highlights the sequence of the motion of a defect present in the triple junction of grains A, B, and C. First, the defect is found to diffuse through the GB between grains A and C. Second, the diffusion length of the defect is approximately one lattice constant from $t=1500$ to $t=2000$ as shown in Figs. $5 \mathrm{a}$ and $5 \mathrm{~b}$, but the diffusion rate varies during the diffusion process. Fig. 5c shows that at $t=6500$ the defect diffuses into the GB between grains $C$ and $D$, where the defect density is higher than that in the former GB. As the defect diffuses away, the lattice distortion in the triple junction alleviates, which promotes the coalescence of grains $\mathrm{A}, \mathrm{B}$, and $\mathrm{C}$ as shown in Fig. 5d. Fig. 6 shows the diffusion process of another defect. This defect diffuses much slower and eventually reaches the right hand edge of the TSV, as shown in Fig. 6c. It is interesting to note that the defect stays still once it reaches the edge of the TSV due to the no-flux boundary condition, which is different from the PFC simulations using a periodic boundary condition [30].

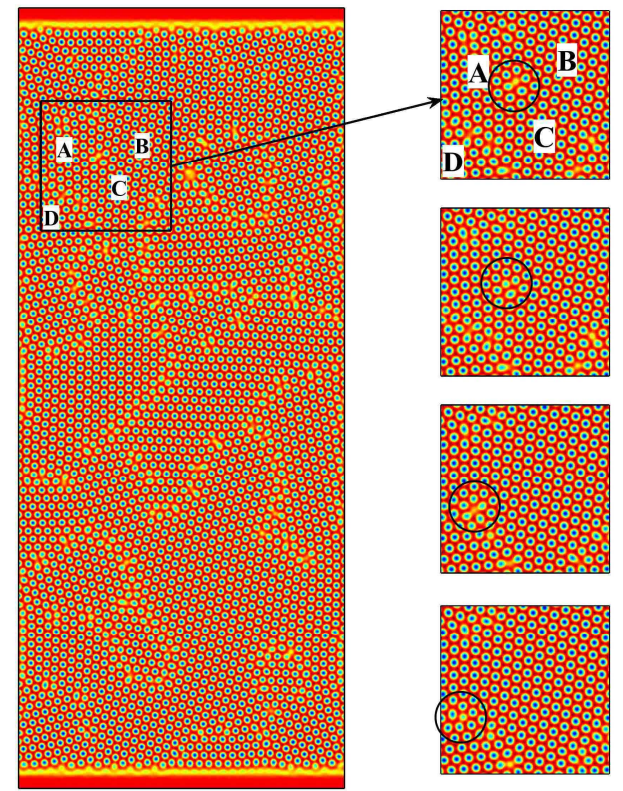

Fig. 5 The process of a defect diffusing from one GB to another. The inserts show the atomistic arrangements at (a) $t=1500$, (b) 2000, (c) 6500, and (d) 7000 .

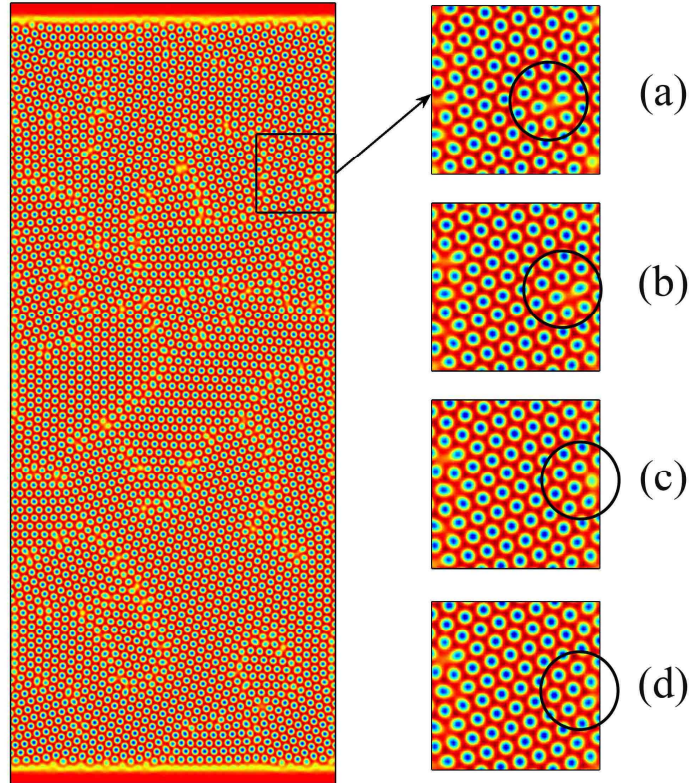

Fig. 6 The process of a defect diffusing to the right edge of the TSV. The inserts show the atomistic arrangements at (a) $t=1000$, (b) 3000, (c) 4000, and (d) 5000 .

The above mentioned two defects both diffuse through the GB network. However, the difference lies in where the network terminates. In fact, the GB network terminates either at the left or right edge of the TSV or at the liquid pools imposed at both the top and bottom of TSVs. As discussed earlier, the defects diffusing through the GB network that terminates at the edges of the TSV stay still, while the defects diffusing through the GB network that terminates at the liquid pools are finally absorbed by the liquid. Therefore, a clustering of defects aggregates at the edges of TSV under shear strain. Note that the liquid pools at the TSV top and bottom are manually imposed for the application of shear strain. It can be expected that defects can also aggregate at both the top and bottom edges of TSVs in real situations where the liquid pools do not exist. Previous work reported that GB sliding and interfacial sliding may cause relative displacement between $\mathrm{Cu}$ and $\mathrm{Si}$ substrate under shear stress [31-33].

\section{Geometry Effect}

The effects of TSV geometry on microstructure and defect motion under shear strain are discussed in this section. The atomistic arrangements in a trapezoid TSV and an hourglass TSV are shown in Fig. 7.

As mentioned earlier, the TSVs of the three geometries have the same initial condition in terms of the configuration of nuclei during the solidification stage, although some of the nuclei may be truncated by the specific geometry. Thus, similarities in the microstructures after the application of shear strain can be found among the three geometries. For example, grain $\mathrm{A}$ in Fig. 7a corresponds to grain $\mathrm{C}$ in Fig. 7c, and grain $\mathrm{B}$ in Fig. 7a corresponds to grain $\mathrm{D}$ in Fig. 7d. Comparing the two TSVs in Figs. 7b and 7d, differences in TSV microstructures under shear strain is observed. Grain A in 
the trapezoid TSV disappears with the shear strain applied, coalesced with other grains around it, yet grain $\mathrm{C}$ in the hourglass TSV still exists. Conversely, grain B in trapezoid TSV remains, while grain D disappears. An explanation to this phenomenon is the length of GB that coincides with the TSV edge. As noted earlier, defects aggregate at the TSV edges and therefore a GB that coincides with the TSV edge is stable. Therefore, the length of GB that resides on the TSV edge is one of the important factors that influences the process of grain coalescence. It is also noted that the curvature of grain $\mathrm{D}$ is larger than grain B and thus disappearing faster in the coalescence process.

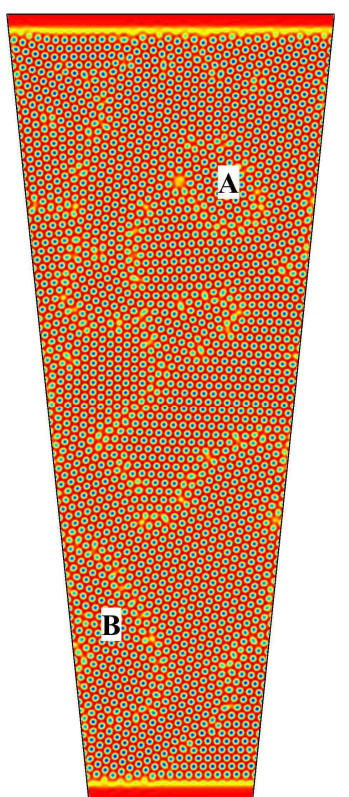

(a)

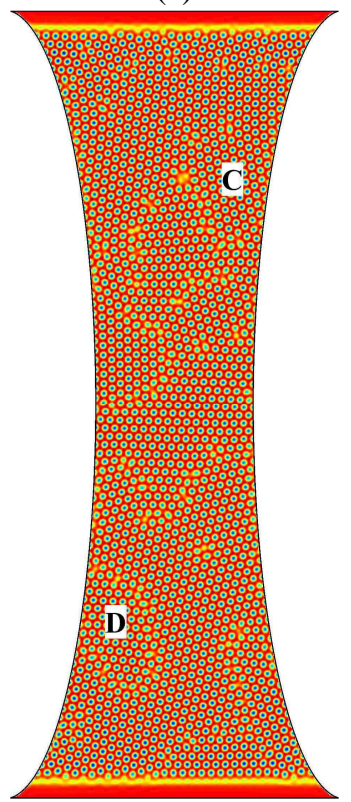

(c)

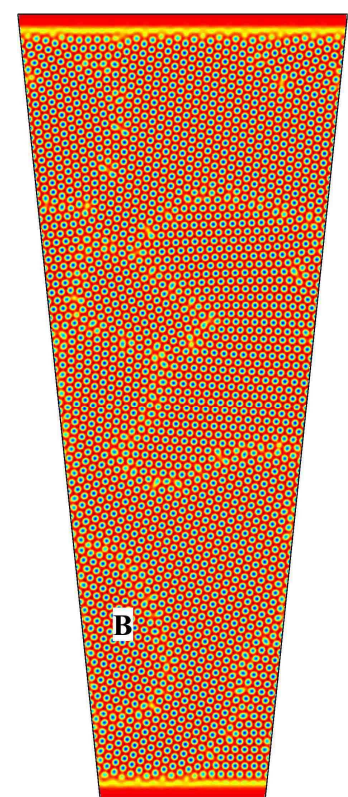

(b)

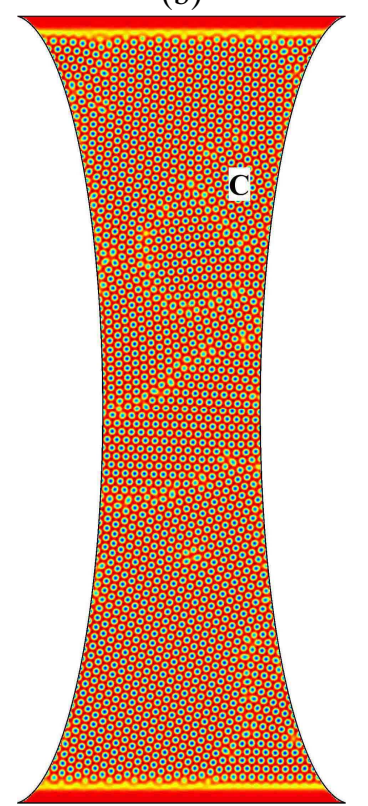

(d)
Fig. 7 Atomistic arrangements in trapezoid TSV at (a) $t=$ 1000 , and (b) 10000, and hourglass TSV at (c) $t=1000$, and (d) 10000.
To further investigate the effects of TSV geometry on defect motion, the simulation results are processed as follows. First, the positions of atoms are identified by the local minimizations of the profile of the order parameter $\rho$. Second, the number of the nearest neighbours of the atoms is counted. If the number of the nearest neighbours is not equal six, the atom is in the vicinity of a GB and its position is recorded. Third, the positions of recorded atoms are compared to their previous locations in the next time step. A position shift of an atom indicates the movement of its neighbouring defects in the GBs. The number of such atoms, hereinafter referred to as AtomS, with a position shift is counted. This number is an index indicating the diffusivity of the defects in the GB. The larger the number, the faster the defects diffuse. Fig. 8 plots the number of AtomS versus time in the three TSVs. At the beginning, a large number of AtomS is found because the density of mobile defects is high. As time goes on, the mobile defects either diffuse to the edges of the TSVs and become immobile, or diffuse to the liquid pools and get absorbed by the liquid. Thus, the number of AtomS decreases with time. Fig. 8 clearly shows that the number of AtomS in the rectangle TSV is the largest among the three geometries, which suggests that the defects diffuse with the fastest rate in the rectangular TSV. A possible explanation is that the ratio between the boundary length and the area of the TSV, i.e. the specific surface area in the case of three dimensions, is what influences the diffusivity of the defects. It is calculated that the ratio of boundary length to area is $0.008,0.011$, and 0.014 for rectangle, trapezoid, and hourglass TSVs, respectively. The larger the ratio is, the higher the density of defects clustering at boundaries, where mobile defects become immobile. Thus, defect motion and GB migration are hindered at the edges of TSVs, leading to more stable microstructures.

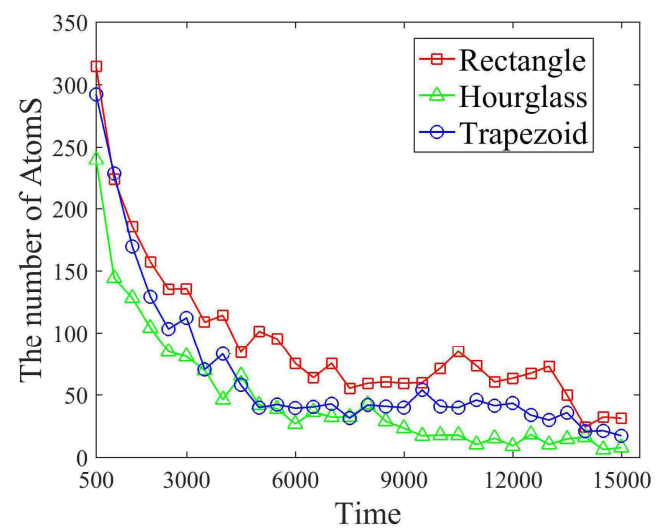

Fig. 8 The plots of the number of AtomS versus time in TSVs with the three different geometries.

\section{Conclusions}

In this study, a PFC model has been employed to investigate the solidification process and microstructural evolution under a shear strain at an atomistic scale in $\mathrm{Cu}$ TSVs of three different geometries. The following conclusions can be drawn:

1. In the solidification process, adjoining grains with a low mis-orientation tend to reorient and coalesce with 
neighbouring grains. The effect of geometry on the solidification rate is not obvious.

2. Under shear strain, accelerated grain rotation and coalescence occur between adjoining grains. Defects diffuse through grain boundaries and aggregate at the edges of the TSVs. Such phenomena may contribute to the $\mathrm{Cu}$ extrusion.

3. The geometry of the TSV is an important factor that influences the behaviour of defect diffusion and microstructures in TSVs, and thus the mechanical behaviour of TSVs.

\section{Acknowledgments}

The authors acknowledge financial support from the Pearl River Science and Technology Nova Program of Guangzhou under grant no. 2012J2200074, the National Natural Science Foundation of China (NSFC) under grant no. 51004118, and Guangdong Natural Science Foundation under grant no. 2015A030312011.

\section{References}

1. Lau J, "Overview and Outlook of Three-Dimensional Integrated Circuit Packaging, Three-Dimensional Si Integration, and Three-Dimensional Integrated Circuit Integration," J Electron Packaging, Vol.136, No.4 (2014), 040801.

2. Lau J, "TSV Manufacturing Yield and Hidden Costs for 3D IC Integration," Proceedings $60^{\text {th }}$ Electronic Components and Technology Conference, Las Vegas, NV, June 2010, pp.1031 - 1042.

3. Okoro $\mathrm{C}$, Vanstreels $\mathrm{K}$, Labie $\mathrm{R}$, Lühn $\mathrm{O}$, Vandevelde B, Verlinden B, Vandepitte D, "Influence of Annealing Conditions on the Mechanical and Microstructural Behavior of Electroplated Cu-TSV," J Micromech. Microeng., Vol.20 (2010), pp.6-11.

4. Jiang T, Wu C, Tamura N, Kunz M, "Study of Stresses and Plasticity in Through-Silicon Via Structures for 3D Interconnects by X-Ray MicroBeam Diffraction," IEEE T. Device. Mat. Re., Vol.14, No.2, (2014), pp.698-703.

5. Athikulwongse K., Chakraborty A, Yang JS, "Stressdriven 3D-IC Placement with TSV Keep-out Zone and Regularity Study," IEEE/ACM International Conference on Computer-Aided Design, San Jose, CA, Nov. 2010, pp.669-674.

6. Stoney GG, "The Tension of Metallic Films Deposited by Electrolysis," Proc $R$ Soc Lond A, Vol.82, No. 553 (1909), pp.172-175.

7. Okoro C, Labie R, Vanstreels K, "Impact of the Electrodeposition Chemistry Used for TSV Filling on the Microstructural and Thermo-mechanical Response of $\mathrm{Cu}, "$ J. Mater. Sci., Vol.46, No.11 (2011), pp. 3868-3882.

8. Jiang T, Ryu SK, Zhao Q, "Measurement and Analysis of Thermal Stresses in 3D Integrated Structures Containing Through-silicon-vias," Microelectron. Reliab., Vol.53, No. 1 (2013), pp.5362.
9. Zhao Q, Im J, Huang R, "Extension of Micro-Raman Spectroscopy for Full-component Stress Characterization of TSV Structures," IEEE $63^{\text {rd }}$ Electronic Components and Technology Conference, Las Vegas, NV, May 2013, pp.397-401.

10. Song M, Mundboth KR, Szpunar J, “Characterization of Local Strain/Stress in Copper Through-silicon Via Structures Using Synchrotron X-ray Microdiffraction, Electron Backscattered Diffraction and Nonlinear Thermomechanical Model," J Micromech. Microeng., Vol. 25, No. 8 (2015), 085002.

11. Vartanian V, Allen RA, Smith L, "Metrology Needs for Through-silicon Via Fabrication," $J$. Micro/Nanolith. MEMS MOEMS, Vol. 13, No.1 (2014), 011206.

12. Radojcic R, Nowak M, Nakamoto M, "TechTuning: Stress Management for 3D Through-silicon-via Stacking Technologies," AIP Conf. Proc., 1378, 5 (2011).

13. Taylor GI, "The Mechanism of Plastic Deformation of Crystals. Part I. Theoretical," Proc. R. Soc. Lond. A, Vol.145 (1934), pp.362-387.

14. Wu Z, Huang Z, Ma Y, "Effects of the Microstructure of Copper Through-silicon Vias on Their Thermally Induced Linear Elastic Mechanical Behavior," Electron. Mater. Lett., Vol.10, No. 1 (2014), pp. 281-292.

15. Nabiollahi N, Moelans N, Gonzalez M, "Microstructure Simulation of Grain Growth in $\mathrm{Cu}$ Through Silicon Vias Using Phase-field Modeling," Microelectron. Reliab. Vol.55, No.5 (2015), pp.765770.

16. Okoro C, Levine LE, Xu R, "Experimentally, How Does Cu TSV Diameter Influence Its Stress State?" IEEE $65^{\text {th }}$ Electronic Components and Technology Conference, San Diego, CA, May 2015, pp.54-58.

17. Raabe D, Ma D, Roters F, "Effects of Initial Orientation, Sample Geometry and Friction on Anisotropy and Crystallographic Orientation Changes in Single Crystal Microcompression Deformation: A Crystal Plasticity Finite Element Study," Acta. Mater., Vol.55, No.13 (2007), pp.4567-4583.

18. Cereceda D, Diehl M, Roters F, "Unraveling the Temperature Dependence of the Yield Strength in Single-crystal Tungsten Using Atomisticallyinformed Crystal Plasticity Calculations," Int. J. Plasticity., Vol. 78(2016), pp.242-265.

19. Elder KR, Katakowski M, Haataja M, "Modeling Elasticity in Crystal Growth," Phys. Rev. Lett., Vol.88 (2002), 245701.

20. Elder KR, Grant M, "Modeling Elastic and Plastic Deformations in Nonequilibrium Processing Using Phase Field Crystals," Phys. Rev. E, Vol.70 (2004), 051605.

21. Granasy L, Podmaniczky F, Toth GI, "Heterogeneous Nucleation of/on Nanoparticles: A Density Functional Study Using the Phase-field 
Crystal Model," Chem. Soc. Rev., Vol.43, No.7 (2014), pp.2159-2173.

22. Elder KR, Provatas N, Berry J, "Phase-field Crystal Modeling and Classical Density Functional Theory of Freezing," Phys. Rev. B., Vol.75, No.6 (2007), 064107.

23. Ofori-Opoku N, Fallah V, Greenwood M, "Multicomponent Phase-field Crystal Model for Structural Transformations in Metal Alloys," Phys. Rev. B, Vol.87, No.13 (2013), pp.2239-2245.

24. Berry J, Provatas N, Rottler J, "Defect Stability in Phase-field Crystal Models: Stacking Faults and Partial Dislocations," Phys. Rev. B, Vol.86, No.22 (2012), pp.278-281.

25. Berry J, Provatas N, Rottler J, "Phase Field Crystal Modeling as A Unified Atomistic Approach to Defect Dynamics," Phys. Rev. B, Vol.89, No.21 (2014), pp.5596-5596.

26. Berry J, Rottler J, Sinclair CW, “Atomistic Study of Diffusion-mediated Plasticity and Creep Using Phase Field Crystal Methods," Phys. Rev. B, Vol.92, No.13 (2015), 134103.

27. Seymour M, Sanches F, Elder KR, "Phase-field Crystal Approach for Modeling the Role of Microstructure in Multiferroic Composite Materials," Phys. Rev. B, Vol.92, No.13 (2015), 184109.

28. Trautt ZT, Adland A, Karma A, Mishin Y, "Coupled Motion of Asymmetrical Tilt Grain Boundaries: Molecular Dynamics and Phase Field Crystal Simulations," Act. Mater., Vol.60 (2012), pp.65286546.

29. Stefanovic P, Haataja M, Provatas N, "Phase Field Crystal Study of Deformation and Plasticity in Nanocrystalline Materials," Phy. Rev. E., Vol.80 (2009), 046107.

30. Hirouchi T, Takaki T, Shibutani Y, Tomita Y, "Development of Numerical Scheme for Phase Field Crystal Deformation Simulation," Comp. Mater. Sci., Vol.44 (2009), pp. 1192-1197.

31. Kumar P, Dutta I, Bakir MS, "Interfacial Effects During Thermal Cycling of Cu-Filled ThroughSilicon Vias (TSV)," J. Electron. Mater., Vol. 41, No. 2 (2012), pp.322-335.

32. Peterson KA, Dutta I, Chen MW, "Diffusionally Accommodated Interfacial Sliding in Metal-silicon Systems," Act. Mater., Vol. 51 (2003), No.10, pp.2831-2846.

33. Wu C, Jiang T, Im J, Huang R, Paul SH, "Effect of $\mathrm{Cu}$ Grain Boundary Sliding on TSV Extrusion," IEEE Electronic Components \& Technology Conference, San Diego, CA, May 2015, pp.661-665. 